\title{
Intraoperative Awareness with Recall Due to Inadvertent Use of Activated Charcoal Filters
}

\author{
Barry N. Swerdlow \\ Oregon Health \& Science University, Portland, OR, USA \\ Email: swerdlow@ohsu.edu
}

How to cite this paper: Swerdlow, B.N. (2019) Intraoperative Awareness with Recall Due to Inadvertent Use of Activated Charcoal Filters. Open Journal of Anesthesiology, 9, 127-132.

https://doi.org/10.4236/ojanes.2019.96012

Received: May 24, 2019

Accepted: June 24, 2019

Published: June 27, 2019

Copyright $\odot 2019$ by author(s) and Scientific Research Publishing Inc. This work is licensed under the Creative Commons Attribution International License (CC BY 4.0).

http://creativecommons.org/licenses/by/4.0/ (c) () Open Access

\begin{abstract}
A 57-year-old woman underwent abdominal surgery with a subarachnoid block supplemented by "light" general endotracheal anesthesia consisting of a propofol infusion and a sub-MAC concentration of sevoflurane. The previous case in the same operating room had involved a malignant hyperthermia-susceptible patient, and charcoal filters had been placed in the breathing circuit as a precautionary measure. Because it had not been used on the evening beforehand, the circuit with filters was left in situ with a strip of tape indicating that it was clean. The woman's anesthesiologist assumed that these filters were heat and moisture exchanger filters in an unused circuit and therefore did not remove them. Subsequently, the patient had awareness with intraoperative recall. This case highlights the potential for inadvertent use of activated charcoal filters with potentially catastrophic results. Such unintended utilization of these products likely can be minimized by improved labeling techniques.
\end{abstract}

\section{Keywords}

Awareness, Charcoal Filters, General Anesthesia, Malignant Hyperthermia, Recall

\section{Introduction}

Awareness with recall (AWR), including both intraoperative consciousness and explicit recall of intraoperative events during general anesthesia, is a potentially devastating occurrence. Prospective, multicenter investigations of AWR report an incidence of $0.1 \%-0.2 \%$ in the general adult population, and risk factors have been identified related to subsets of patients, types of surgeries, anesthetic design, and technical issues [1] [2] [3]. Specifically, total intravenous anesthesia 
(TIVA) has been associated with a higher risk of AWR compared with anesthetics based on volatile agents [4] [5]. As such, particularly when "light" general anesthesia is predicated largely on a propofol infusion, supplementation with measured doses of potent intravenous (IV) amnestics or with a sub-MAC concentration of inhalational anesthesia has been advocated [2].

In this context, an increasingly common, opioid-sparing anesthetic technique for elective major abdominal surgery utilizes a combination of neuraxial blockade and a minimal level of general anesthesia-the latter addition largely to allow for controlled ventilation with adequate surgical relaxation. Because of its many beneficial properties, as part of this scheme, propofol is frequently delivered by infusion often in combination with a sub-MAC dose of volatile agent to minimize the risk of AWR. In such cases, failure to deliver inhalational anesthesia may result in AWR, particularly if relatively low doses of propofol are employed. Some instances of such failure and subsequent AWR have been attributed to equipment failure, including vaporizer and ventilator malfunction [3] [6]. The current report details the occurrence of AWR in a patient otherwise not at high risk for this phenomenon, and highlights an unusual equipment-related etiology for such an adverse event.

\section{Case Description}

A 57-year-old woman [46 kilograms; 1.52 meters] was scheduled for an abdominal sacral colpopexy with a pubovaginal sling to treat symptoms of urinary incontinence associated with vaginal prolapse, a stage III cystocele, and a stage II rectocele. The patient was otherwise in good health without systemic disease, taking no medications, and without a history of substance abuse, including tobacco or alcohol. She had previously undergone general anesthesia on several occasions without AWR.

The patient was the first case of the day in her operating room. When the anesthesiologist performed his pre-anesthesia machine checkout, he noted that the anesthesia circuit had yellow filters in its inspiratory and expiratory limbs. The circuit was "taped" to indicate that it was clean and unused. The filters were labeled "INSP" and "EXP" and had the words "Dynasthetics" and "VAPOR-CLEAN" printed on them in black lettering. The anesthesiologist assumed that these filters were standard heat and moisture exchanger filters (HMEFs), and therefore left them in place. Furthermore, he was unaware that the last surgery in that operating room from the previous evening (performed under monitored anesthesia care) involved an individual with a family history of malignant hyperthermia $(\mathrm{MH})$, and that the previous anesthesiologist had inserted Dynasthetics Vapor-Clean ${ }^{\circledast}$ activated charcoal filters in the circuit as a precautionary maneuver in the event that general anesthesia was necessary. There was no departmental or institutional protocol detailing removal of these filters at the end of $\mathrm{MH}$-susceptible cases.

The patient's initial operating room vital signs included a blood pressure of 
110/50 $\mathrm{mm} \mathrm{Hg}$, a heart rate of 52 beats per minute, and a respiratory rate of 16 breaths per minute. A subarachnoid block was placed without difficulty at L3-4 with $2.0 \mathrm{cc}$ of $0.75 \%$ bupivacaine plus $0.2 \mathrm{mg}$ of Duramorph, resulting in a T6 sensory level. Thereafter, general anesthesia was induced with IV propofol 120 mg and fentanyl $50 \mathrm{ug}$, and an endotracheal tube inserted after neuromuscular blockade (NMB) was achieved with IV atracurium $30 \mathrm{mg}$. Anesthesia was maintained utilizing a propofol infusion at $120 \mathrm{ug} / \mathrm{kg} / \mathrm{min}$ and sevoflurane $0.5 \%$ with a $2 \mathrm{~L} / \mathrm{min}$ oxygen flow; $\mathrm{NMB}$ was maintained via incremental doses of atracurium titrated to $2 / 4$ twitches. The surgery was uneventful, and the patient was hemodynamically stable throughout the operation. Specifically, she did not have tachycardia or hypertension to suggest a sympathetic response. The patient's end-tidal sevoflurane concentration was not recorded, but the anesthesiologist recalls that it was "low." A bispectral index (BIS) monitor was not employed.

The surgery duration was 2 hours and 45 minutes. During closure of the abdomen, with muscle relaxant wearing off, the patient moved her dominant arm to reach for the endotracheal tube. At this point, IV boluses of propofol and midazolam were administered. At the end of surgery, the patient was extubated uneventfully. In the post-anesthesia care unit (PACU), the patient was alert and oriented with stable vital signs and an L1 sensory level.

Shortly after arriving in PACU, the patient related to her health care providers that she had been "awake" during surgery but had been unable to talk; that she had heard operating room speech during the operation but that she had had no pain or discomfort. She recited verbatim phrases that had been spoken at intervals through the procedure. The patient recalled mask preoxygenation, and thereafter her intraoperative memory began shortly after incision: she accurately recalled multiple conversations until the time of her arm movement. The likely cause of her AWR event was explained to the patient and in a postoperative phone call several days later, the patient communicated that she was not upset or significantly troubled by the experience.

\section{Discussion}

Standard recommendations for provision of general anesthesia to $\mathrm{MH}$ susceptible patients involve either use of a "clean" dedicated anesthesia machine-one that has been held in reserve and never exposed to volatile agents, or one from which the vaporizers can be removed and all parts of the machine that absorb significant amounts of volatile anesthetics can be exchanged-or use of a machine that has been flushed with high-flow fresh gas for extended periods in order to achieve an acceptable concentration ( $<5$ parts per million [ppm]) of vapor [7] [8] [9]. Because these methods are labor intense and time-consuming, the use of activated charcoal filters that rapidly reduce volatile anesthetic concentrations to $<5 \mathrm{ppm}$ has become more commonplace [9].

Dynasthetics (Salt Lake City, Utah) manufactures Vapor-Clean ${ }^{\circledast}$ filters that serve this function [7] [9]. Malignant Hyperthermia Association of the United 
States (MHAUS) literature states that these filters effectively reduce vapor concentrations to a level of $<5 \mathrm{ppm}$ for $\geq 12$ hours with fresh gas flows of at least 3 liters/minute [10]. Vapor-Clean ${ }^{\circledR}$ filters are opaque and yellow-orange (or grey) in color. The yellow-orange filters have black writing on the end of the filter that abuts the anesthesia machine; on the grey filters, the writing is white colored. This writing has the following content: "INSP" on one filter and "EXP" on the other, with accompanying arrow symbols to indicate direction of gas flow; "Dynasthetics" and "VAPOR-CLEAN" are written under the words "INSP" and "EXP" (Figure 1).

Vapor-Clean ${ }^{\circledast}$ filters, however, do not have identification on them that clearly distinguishes them from other breathing system filters commonly used in anesthesia circuits, and this lack of clarity can lead to misidentification of the nature of these devices. Specifically, these charcoal filters can be confused with HMEFs that are commonly employed to reduce contamination of the anesthesia machine with bacteria and viruses, as well as cross-infection between patients-a practice that has become increasingly common with the proliferation of multi-drug resistant bacteria [11] [12]. Such an equipment error is made more likely because HMEFs may be located in either or both limbs of the anesthetic circuit (similar to activated charcoal filters) [13], and because many anesthesiologists are unfamiliar with the significance of the company name "Dynasthetics" or the product name "Vapor-Clean". Furthermore, while many HMEFs are colorless and partially transparent, some are colored with varying degrees of opacity.

The present case details the inadvertent use of Vapor-Clean ${ }^{\oplus}$ charcoal filters in a patient receiving a "light" general anesthetic consisting of a relatively low dose propofol infusion supplemented by a sub-MAC concentration of sevoflurane. It is probable that, as a result of these filters, our patient did not receive an adequate dose of volatile anesthesia to prevent AWR. A processed EEG (BIS) monitor (not employed) might have helped avoid this adverse event, and BIS-guided TIVA has been shown significantly to decrease the risk of AWR compared with routine TIVA [5]. Certainly, either BIS or end-tidal anesthesia concentration monitoring are reasonable approaches to avoiding this adverse event in patients receiving heterogeneous anesthetics that include sedative-hypnotic infusions [14]. End-tidal sevoflurane concentration, however, was measured, but no electronic low end-tidal anesthesia concentration (ETAC) alarm was set, and the

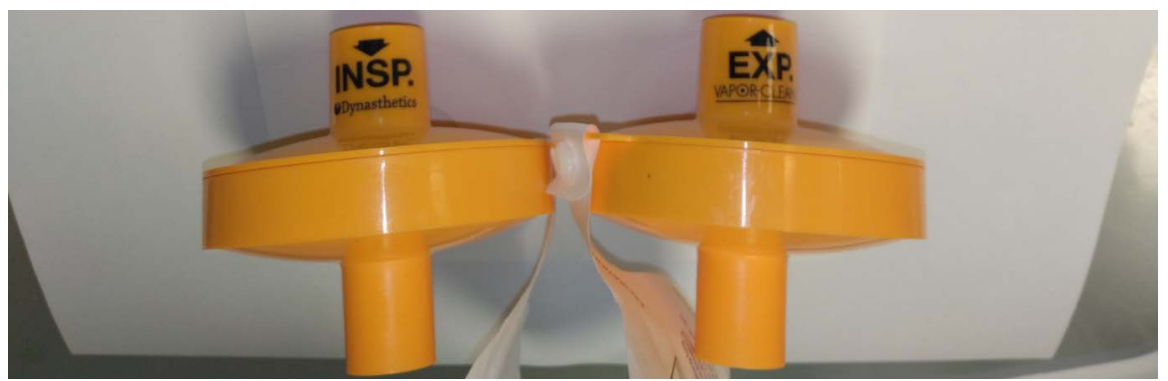

Figure 1. A set of two activated charcoal filters manufactured by Dynasthetics with the brand name Vapor-Clean ${ }^{\oplus}$. 
significance of the low end-tidal value (likely zero) was not appreciated by the anesthesia provider.

AWR has been attributed both to ventilator malfunction and to improper vaporizer calibration [3] [6]. The present case spotlights a different type of equipment issue that can result in the same potentially catastrophic outcome-inadvertent insertion of a charcoal filter or filters in the anesthetic circuit. The anesthesiologist in this report was unfamiliar with the manufacturer's name and product brand name on the filter, and assumed that the devices represented HMEFs. Misidentification of medical supplies due to lack of clear labeling has been repeatedly sited as a root cause of patient-related adverse events [15]. As such, comparable future AWR mishaps may be prevented by adding "FOR MH PATIENTS ONLY" (or similar wording) in bold red lettering to the visible surface of these charcoal filters.

\section{Conclusion}

In conclusion, this report documents a case of AWR associated with inadvertent use of activated charcoal filters in a patient receiving a heterogeneous anesthetic involving a sedative-hypnotic infusion and a low dose of inhalational agent. Possible interventions to prevent recurrence of such an adverse event include use of readily visible labels on these filters-marking them for use with $\mathrm{MH}$ patients only-as well as education of anesthesia technicians and providers regarding the need for removal of the devices at the end of $\mathrm{MH}$-susceptible cases. Although these activated charcoal filters have been marketed in the U.S. since 2010, many anesthesiologists have never used them and do not recognize their unique appearance, and therefore they can easily mistake them for HMEFs. In-servicing of anesthesia personnel at the time of introduction of these filters into institutions would help to correct this suboptimal state of affairs, and-at that same time (i.e. at the time of introduction of the filters) - inclusion in the appropriate departmental and institutional $\mathrm{MH}$ protocols of the need for filter removal after usage ideally should occur. BIS monitoring and use of ETAC alarms are reasonable secondary AWR preventive strategies when TIVA or near-TIVA anesthetic regiments are employed.

\section{Conflicts of Interest}

The author declares no conflicts of interest regarding the publication of this paper.

\section{References}

[1] Apfelbaum, J.L., Arens, J.F., Cole, D.J., et al. (2006) Practice Advisory for Intraoperative Awareness and Brain Function Monitoring. Anesthesiology, 104, 847-864. https://doi.org/10.1097/00000542-200604000-00031

[2] Ghoneim, M. (2000) Awareness during Anesthesia. Anesthesiology, 92, 597-602. https://doi.org/10.1097/00000542-200002000-00043

[3] Hardman, J. and Aitkenhead, A. (2005) Awareness during Anaesthesia. Continuing 
Education in Anaesthesia Critical Care \& Pain, 5, 183-186. https://doi.org/10.1093/bjaceaccp/mki049

[4] Morimoto, Y., Nogami, Y., Harada, K., Tsubokawa, T. and Masui, K. (2011) Awareness during Anesthesia: The Results of a Questionnaire Survey in Japan. Journal of Anesthesia, 25, 72-77. https://doi.org/10.1007/s00540-010-1050-y

[5] Zhang, C., Xu, L., Ma, Y.Q., et al. (2011) Bispectral Index Monitoring Prevent Awareness during Total Intravenous Anesthesia: A Prospective, Randomized, Double-Blinded, Multi-Center Controlled Trial. Chinese Medical Journal, 124, 3664-3669.

[6] Slinger, P.D., Scott, W.A. and Kliffer, A.P. (1990) Intraoperative Awareness Due to Malfunction of a Siemens 900B Ventilator. Canadian Journal of Anesthesia, 37, 258-261. https://doi.org/10.1007/BF03005481

[7] Birgenheier, N., Stoker, R., Westenskow, D. and Orr, J. (2011) Activated Charcoal Effectively Removes Inhaled Anesthetics from Modern Anesthesia Machines. Anesthesia \& Analgesia, 112, 1363-1370. https://doi.org/10.1213/ANE.0b013e318213fad7

[8] Kim, T.W. and Nemergut, M.E. (2011) Preparation of Modern Anesthesia Workstations for Malignant Hyperthermia-Susceptible Patients. Anesthesiology, 114, 205-212. https://doi.org/10.1097/ALN.0b013e3181ee2cb7

[9] Litman, R.S. and Joshi, G.P. (2014) Malignant Hyperthermia in the Ambulatory Surgery Center. How Should We Prepare? Anesthesiology, 120, 1306-1308. https://doi.org/10.1097/ALN.0000000000000256

[10] Malignant Hyperthermia Association of the United States (2011) Preparing the Anesthesia Machine for MHS Patients.

https://www.mhaus.org/healthcare-professionals/be-prepared/preparing-the-anesth esia-machine

[11] Einav, S. and Wiener-Well, Y. (2017) Anesthesia in Patients with Infectious Disease Caused by Multi-Drug Resistant Bacteria. Current Opinions in Anesthesiology, 30, 426-434. https://doi.org/10.1097/ACO.0000000000000457

[12] Chellam, S., Dalal, K.S. and Toal, P.V. (2014) Use of Filters in Anaesthesia: Is It Warranted? Indian Journal of Anaesthesiology, 58, 475-477. https://doi.org/10.4103/0019-5049.139017

[13] Paulsen, A.W. and Klauss, G. (2009) Cross-Contamination via Anesthesia Equipment? APSF Newsletter, 14-15.

[14] Avidan, M.S. and Mashour, G.A. (2013) Prevention of Intraoperative Awareness with Explicit Recall. Anesthesiology, 118, 449-456. https://doi.org/10.1097/ALN.0b013e31827ddd2c

[15] Estock, J.L., Murray, A.W., Mizah, M.T., et al. (2018) Label Design Affects Medication Safety in an Operating Room Crisis: A Controlled Simulation Study. Journal of Patient Safety, 14, 101-106. https://doi.org/10.1097/PTS.0000000000000176 